\title{
STRAIN RECOVERY AND SELF-HEALING IN DUAL CROSS-LINKED NANOPARTICLE NETWORKS
}

\author{
B. Iyer ${ }^{1}$, V. Yashin ${ }^{1}$, T. Kowalewski ${ }^{2}$, K. Matyjaszewski ${ }^{2}$ and A. C. Balazs ${ }^{1}$ \\ ${ }^{1}$ Chemical Engineering Department, University of Pittsburgh, 3700 O'Hara St., Pittsburgh, \\ Pennsylvania 15261, USA - e-mail: bav16@pitt.edu; vvy1@pitt.edu; balazs@pitt.edu \\ ${ }^{2}$ Department of Chemistry, Carnegie Mellon University, 4400 Fifth Ave., Pittsburgh, \\ Pennsylvania 15213, USA - e-mail:tomek@andrew.cmu.edu,km3b@andrew.cmu.edu
}

Keywords: computer simulations, nanoparticle networks, self-healing materials, mechanical recovery

\begin{abstract}
Via computational modeling, we investigate the mechanism of strain recovery in dual cross-linked polymer grafted nanoparticle (PGN) networks. The individual nanoparticles are composed of a rigid spherical core and a corona of grafted polymers that encompass reactive end groups. With the overlap of the coronas on adjacent particles, the reactive end groups form permanent or labile bonds, and thus form a "dual cross-linked" network. We consider the strain recovery of the material after it is allowed to relax from the application of a tensile force. Notably, the existing labile bonds can break and new bonds can form in the course of deformation. Hence, a damaged material could be "rejuvenated" both in terms of the recovery of strain and the number of bonds, if the relaxation occurs over a sufficiently longtime. We show that this rejuvenation depends on the fraction of permanent bonds and strength of labile bonds. Specifically, we show that while an increase in the labile bond energy leads to formation of a tough material, it also leads to delayed strain recovery. Further, we show that an increase in the fraction of permanent bonds yields improved recovery even after multiple stretch-relaxation cycles.
\end{abstract}

\section{INTRODUCTION}

The functionalization of nanoparticles with polymeric chains permits these particles to be cross-linked into extensive networks that can exhibit remarkable mechanical properties [1]. "Dual cross-links", which combine both "permanent" and labile bonds, are known to significantly improve the mechanical properties of polymer gel networks [2]. Here, we use a computational model to study how the dual cross-links affect the elastic and inelastic behavior of polymer grafted nanoparticle (PGN) hybrid materials that are stretched and relaxed (by gradually decreasing the applied force). Simulating the response of these networked PGNs to the application and gradual removal of an applied force is challenging because all the relevant length and time scales should be captured in one specific model. Namely, the model must span a range of architectural features and temporal events. Our recently developed approach [3] encompasses the following components: 1) the essential structural features of the polymer grafted nanoparticles, 2) the interactions between the coated particles, 3) the kinetics of bond formation and rupture between the reactive groups on the polymer chains that form the coating, and 4) the resulting global response of a macroscopic sample to an applied tensile force. 
Building on this multi-component model, we now formulate a procedure for applying a strain-controlled tensile deformation to the material and then a force-controlled release of that deformation. Via this protocol, we specifically determine how the energies of the labile bonds, and the amount of "permanent" bonds in the network affect the recovery of the materials properties and self-healing of the sample after loading and unloading. We also determine how the material behaves after multiple cycles of this strain-controlled deformation and stress-controlled relaxation. Below, we first detail our hybrid, multi-component model.
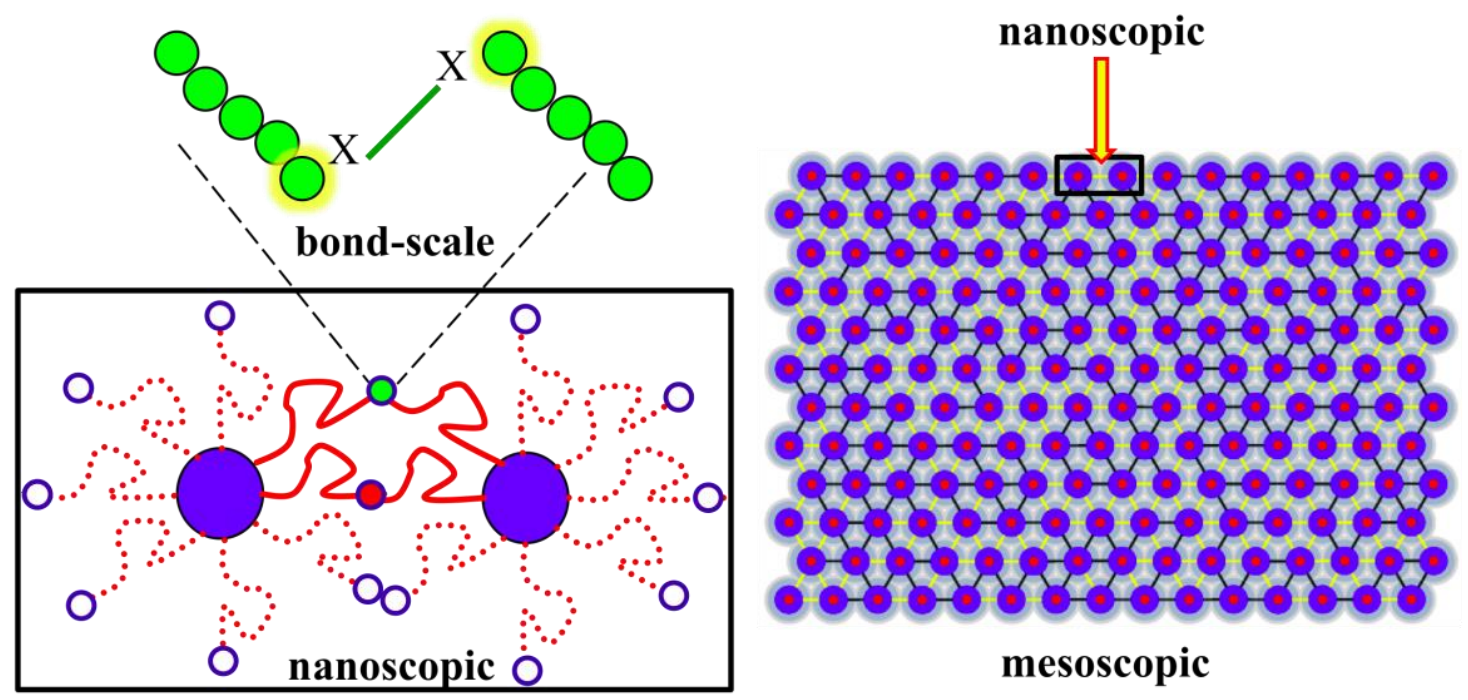

Figure 1: Multiscale interactions in a network of dual cross-linked PGNs.

\section{METHODOLOGY}

Our system consists of a swollen network of cross-linked polymer-grafted spherical nanoparticles (PGNs), which are cross-linked by a combination of "permanent" and labile bonds to form an extended network (see Fig. 1). The center-to-center separation between these coated particles is $R$. The interaction between two such PGNs is modeled through a sum of interaction potentials, which is given by $U_{\text {int }}=U_{\text {rep }}+U_{\text {coh }}+U_{\text {link }}$. The first term characterizes the repulsive interactions between the coated nanoparticles and is given by $[3,4]$ :

$$
\frac{U_{r e p}(R)}{k_{B} T}=\frac{5}{8} f^{3 / 2} \times\left\{\begin{array}{l}
-\ln (R / \sigma)+\left(1+f^{1 / 2} / 2\right)^{-1}, R \leq \sigma \\
\left(1+f^{1 / 2} / 2\right)^{-1}(\sigma / R) \exp \left[-f^{1 / 2}(R-\sigma) / 2 \sigma\right] \quad, R>\sigma
\end{array}\right.
$$

Here, $f$ is the number of arms, and $\sigma=2(1+q)\left(1+2 f^{-1 / 2}\right)^{-1}$ is the range of the potential, which is related to the diameter of the last blob in the Daoud-Cotton (DC) model $[5,6]$. The second term in the potential describes the attractive cohesive interaction between the coated particles and is chosen to have the form:

$$
U_{c o h}(R)=-C\{1+\exp [(R-A) / B]\}^{-1}
$$

where, $C$ is an energy scale, and $A$ and $B$ are length scales [7]. 
The final term in the potential, $U_{\text {link }}$, describes the attractive interaction between particles that are linked by the bonded polymer arms. The attractive force acting between the two bonded particles is given by the following equation [3]:

$$
F_{\text {link }}(r)=N_{b} \kappa(r) r
$$

where, $N_{b}$ is the number of bonds formed between the given pair of particles, and $\kappa(r)$ is the spring constant, which increases progressively with the chain end-to-end distance $r=R-2$ [3]. The stiffening of the chain is described by the following equation obtained for a worm-like chain [8]:

$$
\kappa(r)=\kappa_{0}\left\{1+2\left[1-r^{2}(2 L)^{-2}\right]^{-2}\right\}
$$

In the above equation, $2 L$ is the contour length of the chain formed by bonding two corona arms of length $L$.

The number of polymer cross-links, $N_{b}$, formed between two PGNs by the bonding of the polymer arms depends on the extent of overlap between the coronas of the nanoparticles, and on the kinetics of bond formation and rupture [3]. The set of bonded arms are referred to as a link; in other words, each link in the network corresponds to multiple re-formable bonds. At the individual bond level, we use the Bell model [9] to describe the rupture and re-formation of bonds due to thermal fluctuations. The rupture rate, $k_{r}$, increases exponentially with the applied force $k_{r}=k_{0 r} \exp \left(\gamma_{0} F\right)$, where $k_{0 r}=v \exp \left(-U_{0} / k_{B} T\right)$ is the rupture rate in the zero force limit. The formation rate, $k_{0 f}$, is assumed to be a constant. We note that it is only the labile bonds that can reform after breaking; the permanent bonds can only break. The evolution equation for the number of bonds can be written as [3]:

$$
\frac{d N_{b}}{d t}=-k_{r}(R) N_{b}+P_{c}(R) k_{0 f}\left[N_{\max }(R)-N_{b}\right]^{2}
$$

where $N_{\max }(R)$ is the maximum number of chain ends that could be found in the overlap volume and $P_{c}(R)$ is the probability of contact of two chain ends [3].

The dynamics of the system is assumed to be in the overdamped regime; hence, the motion of each particle described by the equation $d \mathbf{x} / d t=\mu \mathbf{F}_{t o t}$, where $\mu$ is the mobility and $\mathbf{F}_{t o t}$ is the total force on the polymer grafted particle. The total force acting on a particle can be written as $\mathbf{F}_{t o t}=-\partial U_{i n t} / \partial \mathbf{x}+\mathbf{F}_{\text {ext }}$, where $\mathbf{F}_{\text {ext }}$ is the external force acting on the edge particles of the particle array (see Fig 1 ). This equation is solved numerically in two steps since the polymer spring force (within the expression for $\left.F_{t o t}\right)$ in the dynamic equation depends on the number of bonds between particles and consequently, on the evolution of the chemical kinetics given by eq. (5). In the first step, we determine the number of bonds at any given time, $N_{b}(t)$, by evolving numerically the unsteady state kinetics, eq. (5), through an explicit Euler scheme with a time step of $10^{-2} T_{0}$, where $T_{0}$ is the unit of time in the simulation. Note that the numerical evolution of eq. (5) yields a real number, whereas the number of bonds $N_{b}(t)$ should take discrete integer values. In order to determine the integer value, we compare the fractional part of the numerical result, $\left\{N_{b}(t)\right\}$, with a random number $\xi$ 
distributed uniformly between 0 and 1 . If $\left\{N_{b}(t)\right\}<\xi$, then we truncate the result; otherwise, we increment the integer part of the result by 1 . In the second step, we use this value for the number of bonds to calculate the spring force (see eq. (3)) in the dynamic equation and integrate numerically the resulting equation using a fourthorder Runge-Kutta algorithm with a time step of $10^{-2} T_{0}$.

Each sample considered here is composed of $N_{P G N}=180$ particles, which are initially arranged in 12 rows, with 15 particles in each row (see Fig. 1). To quantify the mechanical properties and strain recovery, eight independent runs are performed on samples at each set of model parameters. The strain $\varepsilon$ is calculated as the ratio of the extension of the sample to its length in the undeformed state. Via our computer simulations, we employed a three-step procedure to study the strain recovery of the material (see Fig 2a). In the first step, the sample is subjected to a strain-controlled tensile deformation by pulling the left edge of the sample at a constant velocity of $v=0.001$, which corresponds to roughly $3.55 \mathrm{~nm} / \mathrm{s}$, until the desired maximal value of strain, $\varepsilon_{\max }$, is reached. The values of $\varepsilon_{\max }$ are chosen to not exceed the strain at break. The external tensile force $F$ acting on the left edge of the sample is recorded as a function of $\varepsilon$ during the first step. In the second step, the force $F$ is relaxed to zero at a constant rate over $10^{4} T_{0}$ time steps, and the strain $\varepsilon$ is recorded (forcecontrolled strain relaxation). The inelastic component of the deformation is characterized by the residual strain, $\varepsilon_{0}$, which is recorded at the moment of time when $F=0$. Finally, in the third step of the procedure, the relaxation of the residual strain at zero external force is tracked over some period of time. To elucidate the mechanism of strain recovery, the samples are subjected to either single or multiple cycles of the stretching and relaxation procedure.

\section{RESULTS AND DISCUSSION}

Using the model described above, we determine how the key parameters of the system affect the strain recovery in dual cross-linked networks of PGNs, which are subjected to tensile loading and unloading. We begin by analyzing the effects of varying the energy of the labile bonds, $U_{0}^{(l)}$, on the mechanical response of the material; here, the average number of permanent bonds between a pair of neighboring $P G N s$ was initially set to $P=1$ (i.e., during the initial generation of the sample).

\section{A. Effect of varying the bond energy of the labile bonds}

To obtain insight into the role that the bond energy of the labile bonds plays in the strain recovery process, we consider the deformation of samples with $U_{0}^{(l)}=33,37$ and $39 k_{B} T$. As indicated in Figure $2 \mathrm{a}$, at $0 \leq t \leq 2.5 \times 10^{4} T_{0}$, the samples were stretched at a constant strain rate until the strain reaches the maximal value $\varepsilon_{\max }=0.53$. Then, at $t>2.5 \times 10^{4} T_{0}$, the force $F$ was released at a constant release rate such that $F=0$ at $t=3.5 \times 10^{4} T_{0}$. We use the term "recovery" for the relaxation processes that occur after $F=0$ is reached. From these simulations, we obtain the set of plots in Figure 2, which shows the strain $\varepsilon$ (Fig 2a) and number of labile bonds per particle $N_{b}^{(l)} / N_{P G N}$ (Fig 2b) as a function of time $t$. 

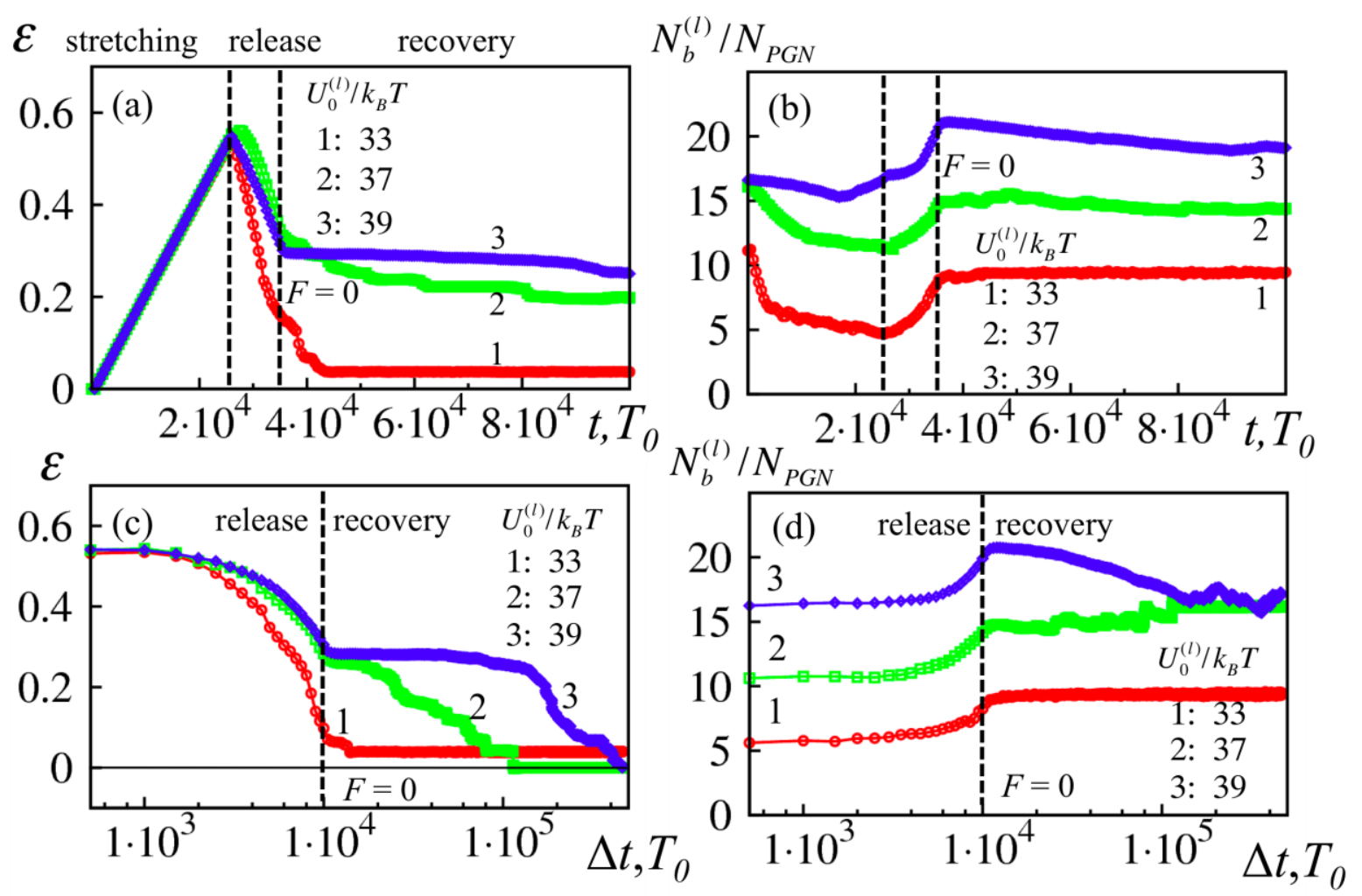

Figure 2: Response of the network to tensile strain applied at a constant velocity and a subsequent controlled, linear decrease of the force. After $F=0$, the sample continues to undergo recovery. (a) Strain $(\varepsilon)$ versus time $(t)$. (b) Number of labile bonds per particle $\left(N_{b}^{(l)} / N_{P G N}\right)$ versus time $(t)$. (c) $(\varepsilon)-(\Delta t)$ curves. (d) $\left(N_{b}^{(l)} / N_{P G N}\right)-(\Delta t)$ curves during release and recovery. The plots are from a single simulation run.

Figure 2 clearly shows that the behavior of the dual cross-linked PGNs depends strongly on the strength of the labile bonds. In particular, Figure 2a indicates that some part of this deformation is inelastic since the samples exhibit residual strain upon release of the external load; the residual strain is considerably lower in the sample having the weaker labile bonds than in the other two samples (compare curve 1 with curves 2 and 3 in Figure 2a). Additionally, Figure 2a shows that the recovery of the residual strain in the unloaded samples at $t>3.5 \times 10^{4} T_{0}$ becomes remarkably slow as the energy of labile bonds increases from $U_{0}^{(l)}=33 k_{B} T$ (curve 1) to 37 and $39 k_{B} T$ (curves 2 and 3 , respectively).

During the course of the deformation, the rupture of stressed labile bonds occurs simultaneously with the formation of new bonds within the overlapped coronas. Figure $2 \mathrm{~b}$ shows that $N_{b}^{(l)} / N_{P G N}$ drops during the initial stretching of a sample, and this decrease slows down even though the stretching continues. The latter behavior indicates that the rupture of stressed bonds is balanced by bond formation. Further, Figure $2 \mathrm{~b}$ shows that owing to the bond formation, the labile bonds are restored in the samples during the release of force $\left(N_{b}^{(l)} / N_{P G N}\right.$ increases at $2.5 \times 10^{4} T_{0}<t<3.5 \times 10^{4} T_{0}$ ), thus, clearly indicating that the material is capable of selfhealing. Figure $2 \mathrm{~b}$ indicates that during the strain controlled stretching there is greater number of the labile bonds in the samples with higher $U_{0}^{(l)}$. 
The relaxation of strain and of the labile bonds continues in the system after the tensile force is released and the system reaches the $F=0$ state. To highlight this behavior, we specifically focus on the time frame around $F=0$ in Figures 2c and 2d. Note that Figure $2 \mathrm{c}$ and $2 \mathrm{~d}$ shows the behavior of the system during the release of force and recovery $\left(t>2.5 \times 10^{4} T_{0}\right)$, as well as the dynamics of recovery at longer times (up to $5 \times 10^{5} T_{0}$ ). From the latter plots, we clearly see that the rate and extent of recovery depend on the energy of the labile bonds. In the system having the weaker labile bonds $\left(U_{0}^{(l)}=33 k_{B} T\right)$, the recovery occurs during $\sim 1 \times 10^{4} T_{0}$ (see Figure $2 \mathrm{c}$ ). The residual strain, however, is not recovered completely and remains about $4 \%$ in this case. Additionally, the number of labile bonds is lower than its initial value (as can be seen by comparing curve 1 at $t=0$ in Figure $2 \mathrm{~b}$ and late times in Figure 2d). In contrast, at the labile bond energies of $U_{0}^{(l)}=37$ and $39 k_{B} T$, the recovery is markedly slower than in the case of weaker bonds and takes about $1 \times 10^{5} T_{0}$ and $5 \times 10^{5} T_{0}$, respectively (see Figure 2c). Furthermore, in these cases, the strain and the number of labile bonds exhibit a complete recovery that occurs in two distinct stages, as can be seen from curves 2 and 3 in Figures $2 c$ and $2 d$, respectively. At the first stage the number of labile bonds relax close to the initial one (i.e., to the one before deformation) while in the second stage strain recovery happens around a constant value of $N_{b}^{(l)} / N_{P G N}$.

Recovery of the residual strain and the labile bonds at $F=0$ and beyond occurs through local rearrangements of the PGNs. The motion of these nanoparticles relative to each other is limited by the rate of breakage of the labile bonds. Note that the labile bonds break less often as the bond energy is increased. Therefore, the rate of recovery decreases with an increase in $U_{0}^{(l)}$. Notably, $U_{0}^{(l)}$ also controls the degradation of the cross-linked system in the course of deformation [3]. An increase in the energy of labile bonds from 33 to $37 k_{B} T$ and higher prevents the formation of large voids, which cannot be healed during recovery. Hence, complete strain recovery is observed at $U_{0}^{(l)}=37$ and $39 k_{B} T$.

\section{B. Effect of varying the fraction of permanent bonds}

Permanent bonds form an elastic skeleton in the dual cross-linked nanoparticle network; the presence of these bonds can lead to notable improvements in the toughness of the material [3]. We anticipate that the permanent bonds between the PGNs will also play an important role in the strain recovery within the sample. To test this hypothesis, we vary the average number of permanent bonds between two particles over the range $1 \leq P \leq 2$, while fixing the labile bond energy at $U_{0}^{(l)}=37 k_{B} T$.

As in the previous section, we examine the behavior of dual cross-linked PGNs that were strained to the maximum extension of $\varepsilon_{\max }=0.53$, and then subjected to the force-controlled relaxation during the period of time $10^{4} T_{0}$, followed by the recovery process. We can obtain insight into the effect of permanent bonds by examining snapshots from simulations of samples in the process of recovery, i.e., after the force in the sample is released. Figures $3 a$ and $3 b$ show the nanoparticles and the labile bonds in the samples at $P=1$ and $P=2$, respectively, at $\Delta t=1.5 \times 10^{4} T_{0}$ and $7.5 \times 10^{4} T_{0}$. Figure 3 a reveals that at $P=1$, there are a number of voids of various sizes distributed throughout the sample during recovery. In contrast, Figure $3 \mathrm{~b}$ shows 
that upon unloading all the voids are healed by $\Delta t=7.5 \times 10^{4} T_{0}$ due to the increase in the number of permanent bonds from $P=1$ to $P=2$.

(a)
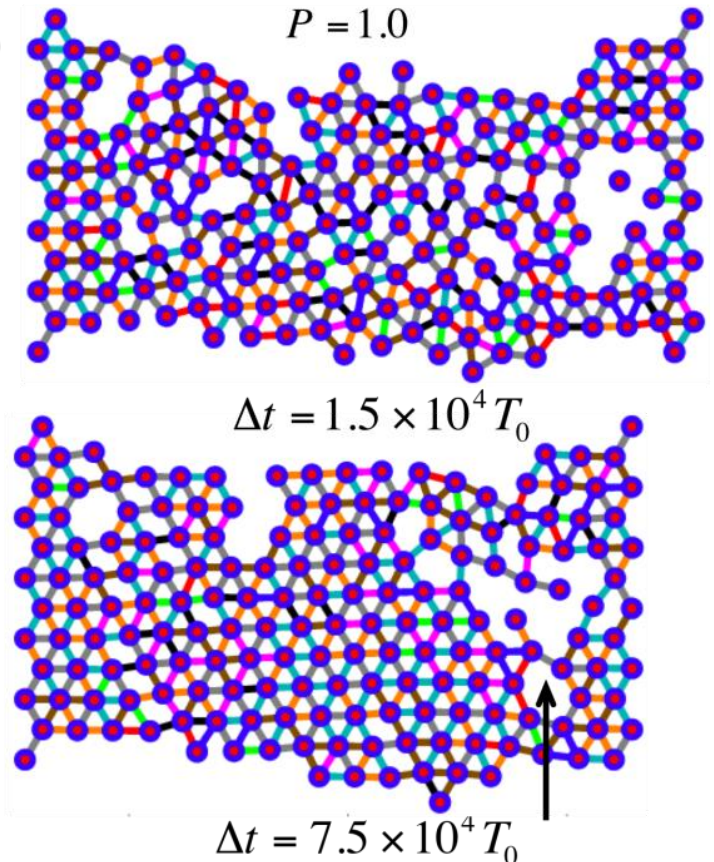

(b)

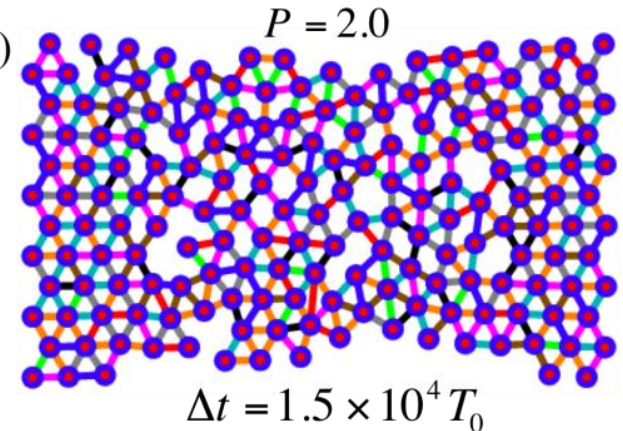

Figure 3: Snap shots of the sample during recovery (a) $P=1$ (b) $P=2$

\section{Effect of applying multiple cycles of stretching and relaxation}

In the above studies, we showed that the structure of the sample could be recovered (rejuvenated) after the forces were released, and that the extent of healing depends on the fraction of the permanent bonds in the network. We now examine the recovery process for systems subjected to multiple cycles of strain and relaxation.

We quantify the influence of $P$ on the properties of materials undergoing multiple deformations by calculating the hysteresis $\Delta W$, which is defined as the area confined within a force-strain loop. Figure 4 shows the material properties of samples that are subjected to repeated deformations where the maximal strain, $\varepsilon_{\max }$, is increased incrementally with each subsequent cycle. Namely, $\varepsilon_{\max }=0.1$ in the first cycle and $\varepsilon_{\max }=0.53$ in the fifth cycle. As above, the consecutive cycles of strain-controlled tensile deformation are followed by the force-controlled relaxation during $10^{4} T_{0}$ and the samples are allowed to recover during the time $5 \times 10^{4} T_{0}$ before the next cycle. Figure $4 \mathrm{a}$ shows the force-strain curves for five consecutive cycles at the fraction of permanent bonds of $P=2$. The curves correspond to a single simulation run at $U_{0}^{(l)}=37 k_{B} T$. While the residual strain increases with an increase in $\varepsilon_{\max }$, the healing of the sample is complete; that is, the strain eventually goes back to $\varepsilon=0$. However, the recovery is incomplete in the $P=1$ sample for strain values $\varepsilon_{\max }>0.31$ and results in modified behavior during the consecutive cycle (see Figure $3 a$ and Figure $4 \mathrm{~b}$ ). In contrast, the sample having more permanent bonds (at $P=2$ ) recovers completely after each cycle (Figure 4a). 
Figure $4 \mathrm{~b}$ shows that the average hysteresis $\Delta W$ (obtained from 8 simulations) increases with an increase in the maximal strain for all samples at $\varepsilon_{\max }<0.42$. Furthermore, while the hysteresis of the samples at $P=2$ is slightly lower than that at $P=1$ at $\varepsilon_{\max }<0.42$ and continues to increase for increasing strains, the hysteresis for the $P=1$ samples levels off at $\varepsilon_{\max }>0.31$. The results indicate that there is higher energy loss with increasing extents of maximum strain due to, in particular, the increase in the residual strain. In addition, at $\varepsilon_{\max } \leq 0.31$, the energy loss is higher at $P=1$ than that at $P=2$. Beyond the latter strain, the deterioration of the sample that occurs at $P=1$ leads to a reduction in $\Delta W$ (see curve 1 in Figure $4 \mathrm{~b}$ ). Thus, an increase in the fraction of permanent bonds is seen to improve recovery.
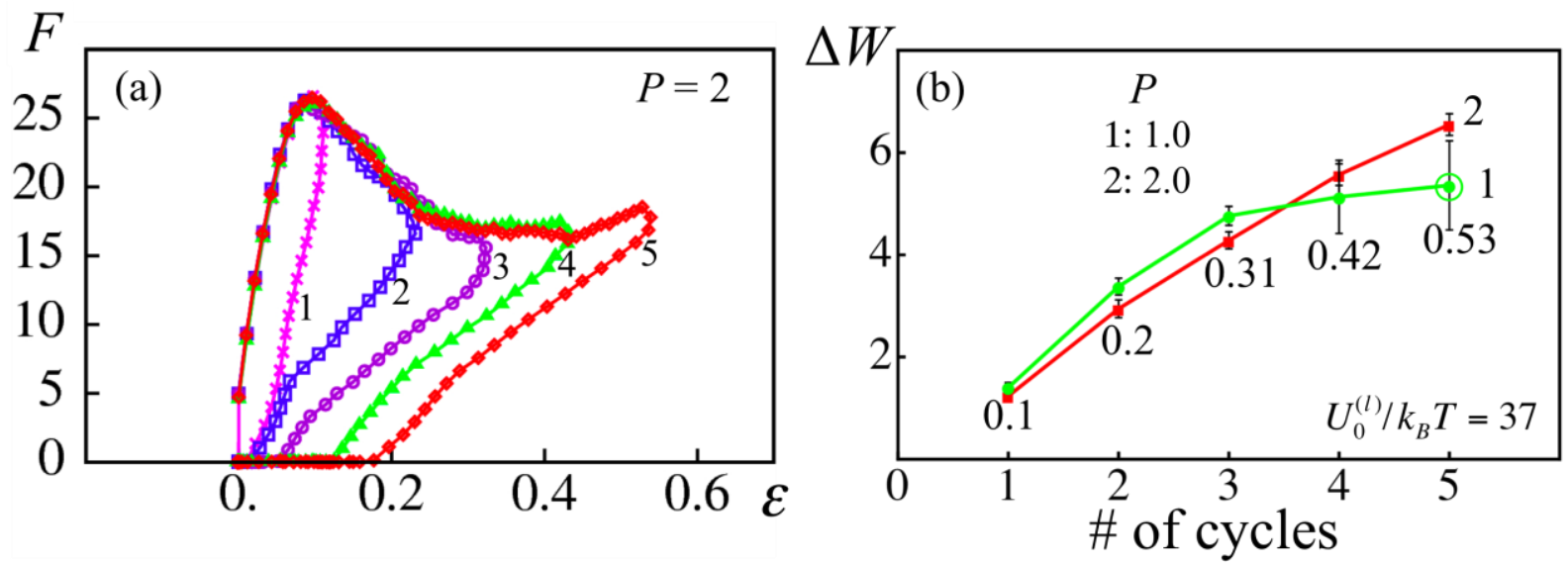

Figure 4: Effect of strain-relaxation cycles on recovery. (a) Force $(F)$-strain $(\varepsilon)$ curves for repeated cycles at $P=2.0$. (b) Hysteresis, $\Delta W$, as a function of number of cycles at $P=1$ and 2 . Open circles indicate conditions when one of the samples fracture.

\section{CONCLUSION}

We examined the strain recovery and healing characteristics of dual cross-linked PGN networks via a hybrid computational model that integrates the kinetics of individual bond rupture/formation with the nano- and meso-scopic structure of the system. Our findings showed that an increase in the energy of the labile bonds led to a decrease in the residual strain within the material. The increase in $U_{0}^{(l)}$ did, however, also lead to an increase in the time scale needed for recovery; this is due to the decrease in the rate of bond breakage with increasing $U_{0}^{(l)}$. These results highlight a competition between the extent and rate of recovery with increasing energies for the labile bonds. For, the studies considered here, the value $U_{0}^{(l)}=37 k_{B} T$ led to an optimal compromise, with the relaxed sample returning to $\varepsilon=0$ on times scales that lay between the $U_{0}^{(l)}=33 k_{B} T$ and $U_{0}^{(l)}=39 k_{B} T$ extremes.

By examining snapshots from the simulations, we found that the $P=2$ networks displayed a more pronounced relaxation of voids within the samples and hence, greater healing than the $P=1$ materials. We also examined the extent of healing and recovery that occurs after the PGN networks were subjected to multiple cycles of stretching and relaxation. Using the hysteresis, $\Delta W$, as a measure of the extent of damage recovery, we found that the $P=1$ materials showed progressive deterioration with consecutive cycles of stretching and relaxation. In contrast, at a higher amount of permanent cross-links $(P=2)$, the networks recovered completely 
after consecutive cycles of stretching and relaxation. Thus, introduction of additional permanent cross-links to the dual cross-linked PGN networks can prevent the effects of pre-stressing by healing defects that were created during deformation unlike filled rubber and thermoplastic elastomers [10].

In summary, these results indicate that the dual cross-linking strategy provides an effective means of designing self-healing PGN networks. The results of these computer simulations reveal how choices in the materials parameters, such as the energy of the labile bonds and fraction of permanent bonds, affect the final mechanical performance of the material.

\section{REFERENCES}

[1] K. E. Mueggenburg, X. M. Lin, R. H. Goldsmith, H. M. Jaeger, Elastic membrane of close-packed nanoparticle arrays, Nature Materials 6 (2007) 656-660.

[2] J. P. Gong, Y. Katsuyama, T. Kurokawa, Y. Osada, Double-network hydrogels with extremely High mechanical strength, Advanced Materials 15 (2003) 1155-1158.

[3] B. V. S. Iyer, I. G. Salib, V. V. Yashin, T. Kowalewski, K. Matyjaszewski, A. C. Balazs, Modeling the response of dual cross-linked nanoparticle networks to mechanical deformation, Soft Matter 9 (2013) 109-121.

[4] C. N. Likos, H. Löwen, M. Watzlawek, B. Abbas, O. Jucknischke, J. Allgaier, D. Richter, Star polymers viewed as ultrasoft colloidal particles, Physical Review Letters 80 (1998) 4450-4453.

[5] A. Jusufi, M. Watzlawek, H. Löwen, Effective interaction between star polymers, Macromolecules 32 (1999) 4470-4473.

[6] M. Daoud, J. P. Cotton, Star shaped polymers: a model for the conformation and its concentration dependence, J. Phys (Paris) 43 (1982) 531-538.

[7] F. Lo Verso, C. N. Likos, L. Reatto, Star polymers with tunable attractions: cluster formation, phase separation, reentrant crystallization, Progress in Colloid Polymer Science 133 (2006) 78-87.

[8] A. V. Dobrynin, Y. J. Carrillo, Universality in nonlinear elasticity of biological and polymeric networks and gels, Macromolecules 44 (2011) 140-146.

[9] G. Diezemann, A. Janshoff, Dynamic force spectroscopy: analysis of reversible bond-breaking dynamics, Journal of Chemical Physics 129 (2008) 0849041-10.

[10] J. E. Mark, B. Erman and F.R. Eirick (Eds.), Science and technology of rubber, 2nd ed., Academic Press, San Diego, 1994. 